\title{
Surface Action Spectroscopy with Rare Gas Messenger Atoms
}

\author{
Zongfang Wu, Agata Płucienik, Yun Liu, Matthias Naschitzki, Walter Wachsmann, Sandy Gewinner, Wieland \\ Schöllkopf, Helmut Kuhlenbeck, ${ }^{\text {a) }}$ and Hans-Joachim Freund \\ Fritz-Haber-Institut der Max-Planck-Gesellschaft, Faradayweg 4-6, 14195 Berlin, \\ Germany
}

(Dated: 24 July 2018)

Action spectroscopy with inert gas messengers is commonly used for the characterization of aggregates in the gas phase. The messengers, often rare gas atoms or $\mathrm{D}_{2}$ molecules, are attached to the gas phase aggregates at low temperature. Vibrational spectra of the aggregates are measured via detection of inert gas desorption following a vibrational excitation by variable-energy infrared light. We have constructed an apparatus for the application of action spectroscopy to surfaces of solids with the aim of establishing a new method for the vibrational spectroscopy of surfaces and deposited clusters. Experiments performed for neon covered $\mathrm{V}_{2} \mathrm{O}_{3}(0001)$ show that this method can provide information about surface vibrations. Besides the surface sensitive channel there is also a bulk sensitive one as demonstrated with the example of $\mathrm{CeO}_{2}(111)$ thin film data. Unlike IRAS, normalization to a reference spectrum is not required for action spectroscopy data and unlike HREELS, the action spectroscopy method does not suffer from moderate resolution nor from multiple excitations. Selective decoration of specific surface features with messenger atoms may be utilized to focus the spectroscopic information onto these features.

\section{INTRODUCTION}

Action spectroscopy with inert gas messenger atoms is a method to obtain vibrational spectra of gas phase aggregates $^{1-16}$. Inert gases are characterized by a weak interaction with the aggregates. Consequently they modify the aggregate properties only weakly and the bond to the aggregates may be cleaved with little energy. Such inert gas atoms or molecules are attached to the aggregates at low temperature and the desorption by sufficiently intense infrared irradiation is monitored as a function of the photon energy. Desorption may occur when the infrared light excites a vibration in the clusters and therefore a plot of the inert gas desorption rate or the production rate of clusters with missing inert gas atoms/molecules as a function of the photon energy represents a vibrational spectrum. This role in the measuring process is why the attached inert gas atoms/molecules are called messengers. The vibrational spectrum of the gas phase aggregates is often used to identify their structure via comparison with computed vibrational spectra of different model structures ${ }^{3,5,8}$.

Model catalysis is a branch of science, which studies simplified models of catalysts, often consisting of clusters deposited on an oxidic single crystal or an ordered oxide film, with the aim of deepening the understanding of catalytic processes at surfaces ${ }^{17}$. The size and the structure of the deposited clusters are very relevant parameters for the catalytic processes at the surface and therefore it would be important to know details about the cluster structure. However, this information is not easily accessible. Scanning probe microscopy permits to get information about the cluster surface, if atomic resolution can be obtained at all, while the structure below

\footnotetext{
a)Electronic mail: kuhlenbeck@fhi-berlin.mpg.de
}

the surface remains hidden. Photoelectron diffraction and EXAFS (extended X-ray absorption fine structure) might reveal a partial set of information, while vibrational methods like IRAS (infrared reflection absorption spectroscopy) and HREELS (high resolution electron energy loss spectroscopy) may not be applicable at all due to the small excitation cross section of metal cluster vibrations. TEM (transmission electron microscopy) may disclose a reasonable set of details about the structure of deposited clusters for systems suitable for this method.

We have recently shown that the action spectroscopy method can also be applied to surfaces covered with a layer of rare gas messenger atoms ${ }^{18}$. For these studies we have set up an experiment, which uses light from the infrared free electron laser (FEL) of the Fritz Haber Institute (FHI). This device is well suited as a radiation source for the surface action spectroscopy experiment thanks to its high beam intensity and its wide range of available photon energies for a given set of FEL parameters.

Surface action spectroscopy extends the set of vibrational methods applicable to surfaces by a new member. In this publication we will present two examples for the application of this method. A surface action spectrum of $\mathrm{CeO}_{2}(111) / \mathrm{Ru}(0001)$ is discussed as an example for bulk sensitive spectroscopy while spectra of $\mathrm{V}_{2} \mathrm{O}_{3}(0001) / \mathrm{Au}(111)$ contain also bands from surfacelocated vibrations. In the surface-sensitive channel this method reveals information only from surface areas covered by the inert gas atoms. Therefore selective decoration of deposited clusters with inert gas atoms might be a way to focus the spectroscopic eye on just these components. Other scientific topics where action spectroscopy of surfaces might be useful are surface vibrations, surface defects and also excitations in the bulk. 


\section{EXPERIMENTAL SETUP}

Figure 1 displays a drawing of the experimental setup. The central components are a liquid helium cooled flow cryostat, which cools the sample holder with the sample, and a quadrupole mass spectrometer (QMS) from Extrel (Extrel MAX 500 HT) for detection of the desorbing inert gas atoms. For characterization of the surface structure a low-temperature AFM/STM system is available. Most of the experiments were performed with neon messenger atoms, in one case also argon was employed. Therefore the following discussion will focus on rare gas messenger atoms.

The QMS is mounted in a pumped housing with a small opening ( $4 \mathrm{~mm}$ diameter) through which the rare gas atoms desorbing from the sample surface can enter for detection by the QMS. Therefore the sample is positioned in front of the opening at a distance of a few millimeters during the experiments. A K-type (ChromelAlumel) thermopair was spot-welded to the sample for temperature measurement. The thermocouple performs reasonably well at the high temperatures required for sample preparation, but has a significant level of inaccuracy at the very low temperatures required for the action spectroscopy experiments. Therefore the sample temperature during the action spectroscopy experiments is not known accurately, but it is clear that it was too high for neon multilayer formation (less than $\sim 6 \mathrm{~K})^{19}$ and too low for neon monolayer desorption (above $\sim 10 \mathrm{~K}$ for $\left.\mathrm{Ru}(0001)^{19}\right)$ in the experiments discussed here.

In order to reduce the infrared radiation power reaching the sample from the surrounding (chamber walls, QMS housing, etc...), the QMS housing is cooled with liquid nitrogen. This additionally reduces the chamber pressure and the pressure in the QMS housing, which is relevant since at the low sample temperatures reached during the experiments every type of gas atoms/molecules (except helium atoms) adsorbs at the surface. A further reduction of the infrared radiation power was achieved by blocking the direct view of the sample to the filament of the mass spectrometer with two cold metal sheets (see Figure 2). Rare gas dosing was performed by filling the QMS housing with the rare gas while the sample was positioned in front of the opening in the QMS housing. A computer controlled piezo dosing valve was used to adjust the gas flow.

The infrared beam enters the chamber through a cesium iodide window, illuminating the sample surface at an angle of $70-83^{\circ}$ with respect to the surface normal (see Figure 3). At such a grazing angle the distance between the opening of the QMS housing and the sample can be rather small and the infrared reflectivity will be large for metal samples, which is profitable since it limits the heat load of the sample. The area on the surface illuminated by the beam had a diameter of a few millimeters. A signal approximately proportional to the infrared beam power was provided by a type $K$ thermocouple coated with an infrared absorbing layer. This sensor was located in the

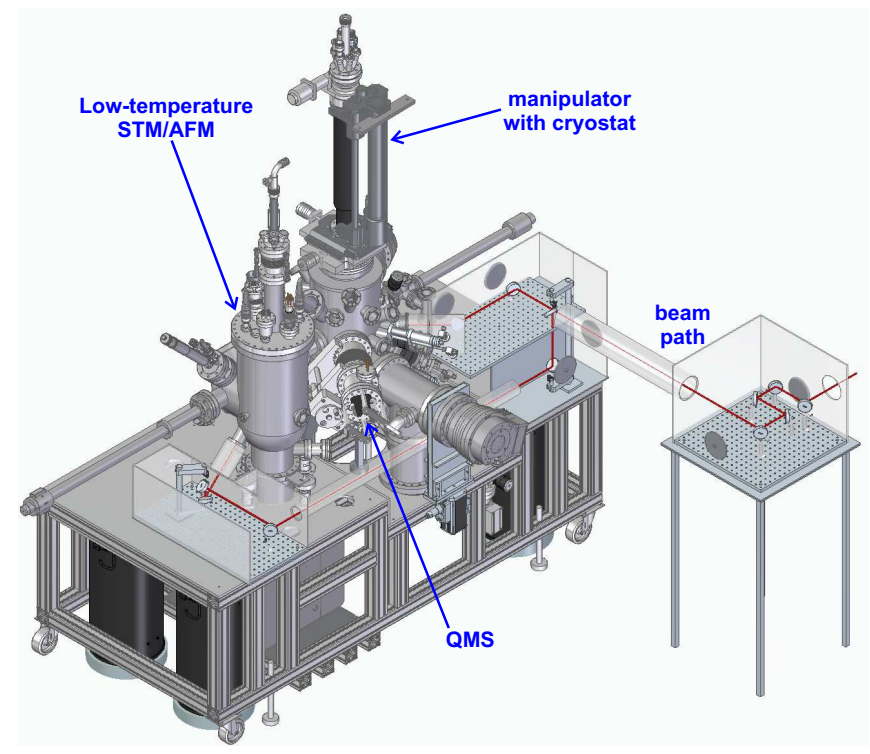

FIG. 1. (color online). Drawing of the experimental setup used for the surface action spectroscopy experiment.

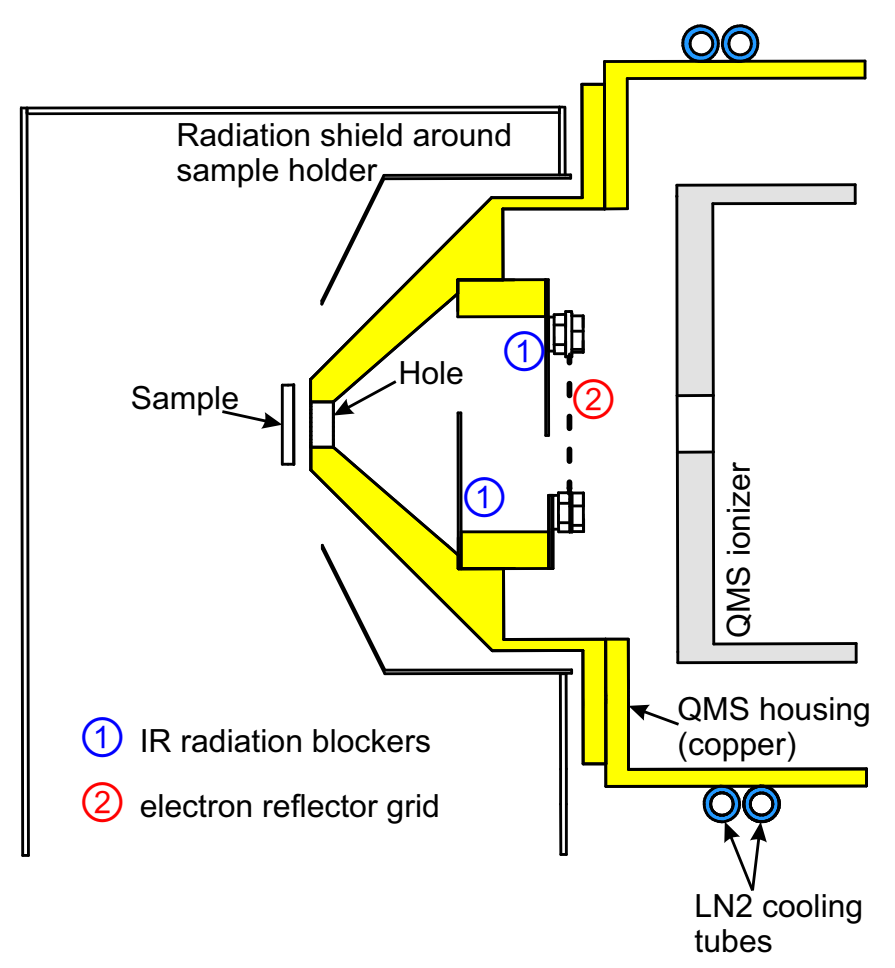

FIG. 2. (color online). Drawing of the QMS housing, side view.

IR beam path between the cesium iodide window and the sample where it was warmed up by the infrared radiation.

The FHI FEL generates macro pulses ( 5 to $8 \mu$ s long pulse trains at 5 or $10 \mathrm{~Hz}$ repetition rate) consisting of some thousand short pulses ('micro pulses') at a rate of $1 \mathrm{GHz}$. Each of them has a duration of a few picoseconds ${ }^{20}$. The energy per micro pulse is $\sim 10 \mu \mathrm{J}$, cor- 


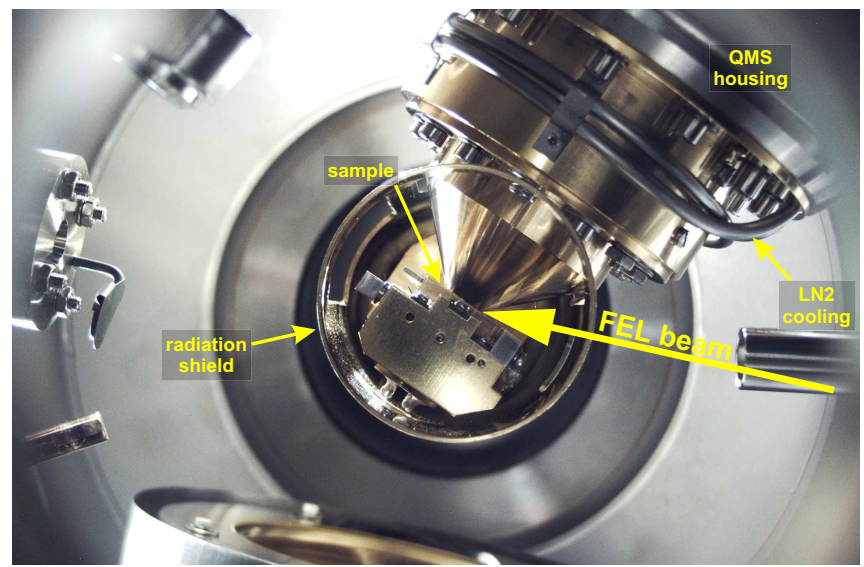

FIG. 3. (color online). Photograph of the sample on the sample holder in front of the quadrupole mass spectrometer housing (taken from the chamber bottom).

responding to $\sim 50-80 \mathrm{~mJ}$ per macro pulse. The spectral width of the radiation is about $0.5 \%$ (full width at half maximum) of its central wavelength ${ }^{20}$.

\section{SAMPLE PREPARATION}

The $\mathrm{CeO}_{2}(111)$ and $\mathrm{V}_{2} \mathrm{O}_{3}(0001)$ thin films have been prepared according to published recipes ${ }^{21-26}$. Metal was deposited in all cases with electron beam evaporators manufactured by Focus, Germany. Vanadium was evaporated by direct electron beam heating of a $2 \mathrm{~mm}$ thick vanadium wire, while cerium was evaporated out of a crucible. The deposition rate of the evaporators was calibrated with a quartz microbalance before metal deposition onto the sample.

The $\mathrm{V}_{2} \mathrm{O}_{3}$ (0001) film on $\mathrm{Au}(111)$ was prepared by evaporation of metallic vanadium (using an Focus EFM3 electron beam evaporator) in an oxygen atmosphere $\left(1 \times 10^{-7} \mathrm{mbar}\right)$ at $600 \mathrm{~K}$ followed by annealing at $670 \mathrm{~K}$ in $1 \times 10^{-7}$ mbar of oxygen and annealing in vacuum at $850 \mathrm{~K}$. Evaporation rates between 0.5 and $1 \AA / \mathrm{min}$ were employed in the experiments. The prepared $\mathrm{V}_{2} \mathrm{O}_{3}(0001)$ films were usually about $10 \mathrm{~nm}$ thick.

$\mathrm{CeO}_{2}$ (111) films were grown on $\mathrm{Ru}(0001)$ with a $(2 \times 2)$ oxygen overlayer using a recipe adopted from Mullins et $\mathrm{al}^{25,26}$ and $\mathrm{Lu}$ et $\mathrm{al}^{24}$. Rather granular layers are formed due to the $\sim 40 \%$ mismatch between the lattice parameters of the $\mathrm{Ru}(0001)$ substrate and the $\mathrm{CeO}_{2}(111)$ overlayer if the layer is grown according to the standard procedure by deposition of cerium in an oxygen ambient atmosphere at elevated temperature ${ }^{27}$. Therefore, we prepared a kinetically hindered oxide interlayer on $(2 \times 2) \mathrm{O} / \mathrm{Ru}(0001)$ by deposition of $7 \AA$ cerium in $1 \times 10^{-6}$ mbar of $\mathrm{O}_{2}$ while ramping the temperature with a rate of $1 \mathrm{~K} / \mathrm{s}$ from $100 \mathrm{~K}$ to $670 \mathrm{~K}$. In the final preparation step the temperature was kept at $670 \mathrm{~K}$ during deposition of the rest of the layer in $1 \times 10^{-6} \mathrm{mbar}$ of
$\mathrm{O}_{2}$, followed by oxidation at $\sim 980 \mathrm{~K}$ at the same oxygen pressure. The thickness of the prepared $\mathrm{CeO}_{2}(111)$ layer was $5 \mathrm{~nm}$.

\section{THE METHOD, SOME CONSIDERATIONS}

In action spectroscopy experiments gas phase clusters absorb energy from an infrared beam until they break into fragments. The fragmentation rate as a function of the photon energy is the output of the experiment. The sensitivity of the method may be increased by attaching inert gas atoms (the messengers) to the clusters. Desorption of such atoms requires only little energy: a few photons or even just a single one may suffice to induce desorption (which is the fragmentation process in this case). Rare gas atoms are often used since they affect the properties of the clusters only weakly - the lighter the rare gas atoms the weaker the bond and the smaller the influence on the cluster properties.

A comparable process is conceivable also for dielectric solids and thin dielectric films with a layer of adsorbed rare gas atoms. In this case essentially all non-reflected intensity of the infrared beam may be absorbed and the sample may warm up to temperatures above the rare gas desorption temperature. The FHI free electron laser reaches a time-averaged power in the range of $1 \mathrm{Watt}^{20}$, which can warm up a cooled sample by a few Kelvin. This temperature rise will induce adsorbate desorption if the sample temperature before laser illumination is not too low so that a temperature above the rare gas desorption temperature can be reached by the illumination. Bulk vibrations can absorb a substantial part of the infrared power and therefore a significant temperature rise may be expected. Adsorbate layers with monolayer or submonolayer coverage or microscopic vibrational surface states absorb at most a few percent of the infrared beam power (see for instance ${ }^{28,29}$ ). Consequently the temperature rise will be much smaller in this case and it depends on the studied system whether absorption of infrared radiation can induce rare gas desorption or not. The two limiting cases of rare gas desorption will be discussed in the following:

1. In the thermal channel the energy uptake is high and thermalization leads to a substantial sample temperature rise, which eventually may induce rare gas desorption. This channel will dominate for vibrations with a high absorption cross section like bulk polaritons. Here the energy is mostly absorbed in the bulk and a transfer of energy to the surface is required as an intermediate step. This is why this channel may also be called indirect channel.

2. Surface vibrations are excited by the infrared light in the direct coupling channel. In this case the energy uptake rate will be small and so will 
be the time and position averaged sample temperature rise. Nevertheless, the rare gas atoms may still desorb if the energy dissipation channels guide enough energy into the bond of the rare gas atoms to the surface. A weak coupling of the vibration to the bulk (resulting in a high lifetime) may be beneficial since this may prevent the energy from diffusing too quickly into the bulk.

Some additional complexity comes from the time structure of the FEL beam as will be discussed below.

\section{EXPERIMENTAL RESULTS}

\section{A. The thermal channel- $\mathrm{CeO}_{2}(111) / \mathrm{Ru}(0001)$}

Data for $\sim 5 \mathrm{~nm} \mathrm{Ce}_{2} \mathrm{O}(111)$ on $\mathrm{Ru}(0001)$ with a neon messenger layer are shown in Figure 4. The blue curve is the sample temperature rise resulting from absorption of FEL infrared light. There is a significant level of noise, which is due to the small temperature change and the use of a Chromel-Alumel thermopair, which does not perform very well at these low temperatures. Nevertheless, an asymmetric temperature maximum at $\sim 600 \mathrm{~cm}^{-1}$ is clearly detectable. The maximum in the temperature rise curve and the action spectrum are at very similar energies indicating that rare gas desorption may be caused by thermal effects. The temperature increase is due to absorption of light from the FEL beam, with the heating power being the power of the incident beam minus the power of the reflected beam. Reflectance data in combination with energy dependent FEL beam power data contain all the information required to calculate the heat load. Non-linear optical processes are not relevant under the given conditions and can therefore be ignored.

We have performed a calculation of the heat load using dielectric theory. Optical data for the infrared region have been published by Marabelli and Wachter ${ }^{30}$ and Mochizuki and Tateyama ${ }^{31}$. Mochizuki ${ }^{32}$ discusses a thin film of $\mathrm{CeO}_{2}$. We have used the data of Mochizuki and Tateyama ${ }^{31}$ for the calculation of the reflectance since the peak width in the computed reflectance spectrum fits better to our measured data than the one resulting from computations using the data of Marabelli and Wachter ${ }^{30}$. Mochizuki and Tateyama report transversal optical phonon (TO) modes $\omega_{1}^{T O}=272 \mathrm{~cm}^{-1}$ and $\omega_{2}^{T O}=424 \mathrm{~cm}^{-1}$ with damping parameters $\Gamma_{1} / \omega_{1}^{T O}=0.059$ and $\Gamma_{2} / \omega_{2}^{T O}=0.26$ and oscillator strengths $4 \pi \varrho_{1}=18.9$ and $4 \pi \varrho_{2}=0.336^{31}$. Reported static and high-frequency dielectric constants are $\epsilon_{0}=24.5$ and $\epsilon_{\infty}=5.31^{31}$. With these number the complex dielectric function was formulated as a sum of damped Lorentzian oscillators:

$$
\epsilon_{C e O 2}(\omega)=\epsilon_{\infty}+\sum_{j=1}^{2} \frac{4 \pi \varrho_{j} \times\left(\omega_{j}^{T O}\right)^{2}}{\left(\omega_{j}^{T O}\right)^{2}-\omega^{2}+i \omega \Gamma_{j}}
$$

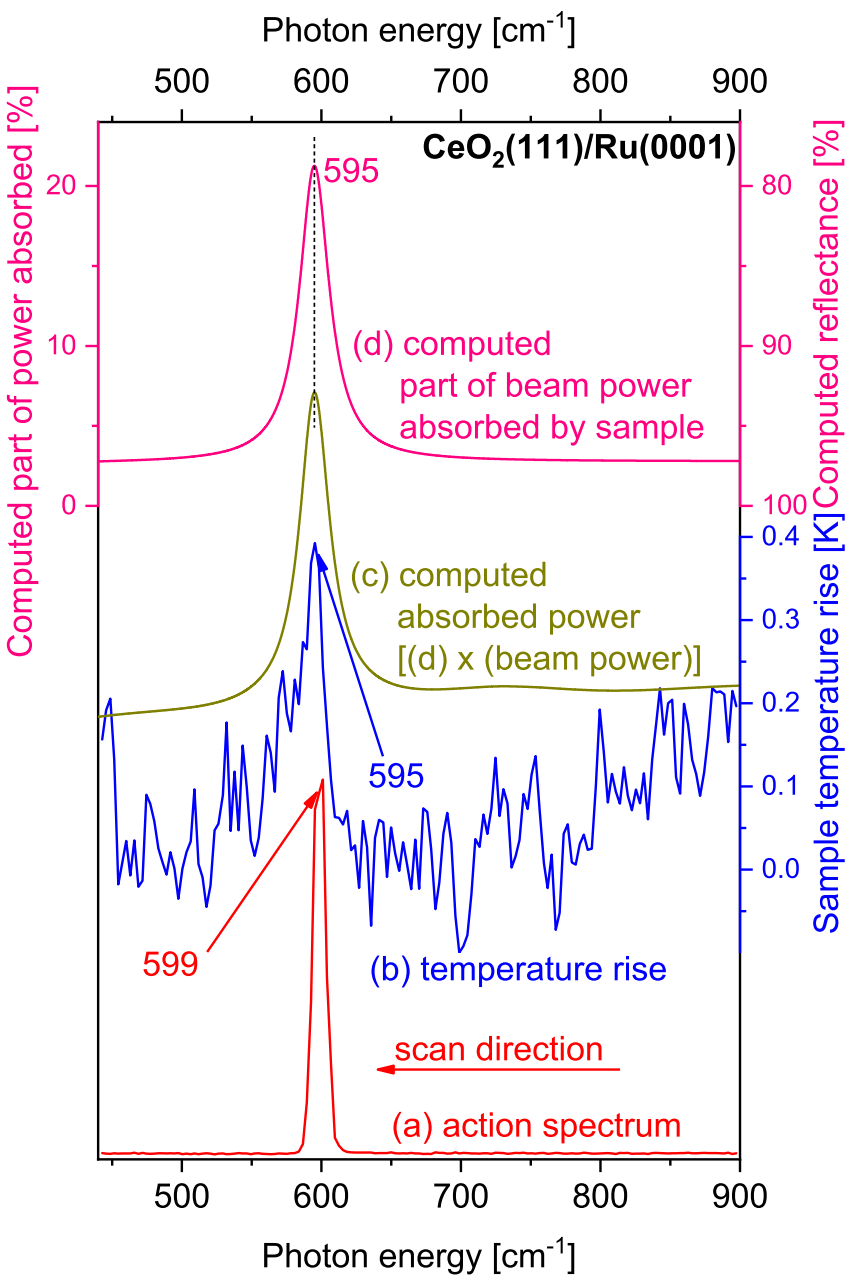

FIG. 4. (color online). Surface action spectrum [red curve, (a)] of a neon covered $\mathrm{CeO}_{2}(111) / \mathrm{Ru}(0001)$ thin film. The sample temperature rise (b) is shown in blue. The pink curve (d) displays the computed part of the beam power absorbed by the sample and the green curve (c) is curve (d) multiplied by the FEL beam power (see supplemental material).

where $\omega$ is the angular frequency of the incident light. The calculation of the reflectance of the thin film on $\mathrm{Ru}(0001)$ requires also the dielectric function of the ruthenium substrate. This was modeled with the Drude model

$$
\epsilon_{R u}(\omega)=1-\frac{\omega_{P}^{2}}{\omega^{2}+i \omega \Gamma_{R u}}
$$

$\omega_{P}$, the ruthenium plasmon angular frequency, was computed from the valence electron density $n_{e}$ of ruthenium: $\omega_{P}^{2}=n_{e} q_{e}^{2} /\left(m_{e} \epsilon_{0}\right)$, with $n_{e}$ the valence electron density, $q_{e}$ the electron charge, $m_{e}$ the electron mass, and $\epsilon_{0}$ the vacuum permittivity. This gave a plasmon energy of $28.5 \mathrm{eV}$, which is not far from $30 \mathrm{eV}$ mentioned by Frederick et $\mathrm{al}^{33}$. The damping constant $\Gamma_{R u}$ was set to $0.05 \mathrm{eV}$, which is actually a value for gold ${ }^{34}$ since a value for ruthenium could not be found. Also, the uniaxial nature of the ruthenium hcp crystal structure is not con- 
sidered by this treatment. These neglects and approximations have a limited influence on the energetic position of the structure in the reflectance spectrum but affect the absolute reflectance numbers to some extent.

Equations for the calculation of the reflectance of a thin layer may be found in the book of Born and Wolf ${ }^{35}$, see also Heavens ${ }^{36}$. In the following discussion medium 1 is the vacuum with $\epsilon_{1}=n_{1}^{2}=1$, medium 2 the $\mathrm{CeO}_{2}(111)$ film with $\epsilon_{2}(\omega)=n_{2}(\omega)^{2}=\epsilon_{\mathrm{CeO} 2}(\omega)$ and medium 3 the $\operatorname{Ru}(0001)$ substrate with $\epsilon_{3}(\omega)=n_{3}(\omega)^{2}=\epsilon_{R u}(\omega)$. The $n_{j}$ are the complex refractive indices. With $\mathrm{r}_{12}(\omega)$ and $\mathrm{r}_{23}(\omega)$, the amplitude reflectivities at the interfaces between medium 1 and 2 and medium 2 and 3, respectively, the amplitude reflectivity of the thin film system may be written as:

$$
r(\omega)=\frac{r_{12}(\omega)+r_{23}(\omega) \xi(\omega)}{1+r_{12}(\omega) r_{23}(\omega) \xi(\omega)}
$$

with $\xi(\omega)=\exp (2 i \beta(\omega)), \quad \beta(\omega)=$ $n_{2}(\omega) d_{C e O 2} \cos \left(\phi_{2}(\omega)\right) \omega / c, \quad d_{C e O 2}$ the thickness of the $\mathrm{CeO}_{2}(111)$ layer and $c$ the speed of light. $\phi_{2}(\omega)$ is the angle of the light wave relative to the surface normal in the $\mathrm{CeO}_{2}(111)$ film and can be calculated with Snell's law:

$$
n_{1} \sin \left(\phi_{1}\right)=n_{2}(\omega) \sin \left(\phi_{2}(\omega)\right)
$$

$\phi_{1}$, the light incidence angle was $83^{\circ}$ in the experiment discussed here. The reflectivities $\mathrm{r}_{12}(\omega)$ and $\mathrm{r}_{23}(\omega)$ can be calculated with the Fresnel equations. Since the light was p-polarized, the Fresnel equations for p-polarized light had to be used:

$$
r_{x y}(\omega)=\frac{n_{y}(\omega) \cos \phi_{x}(\omega)-n_{x}(\omega) \cos \phi_{y}(\omega)}{n_{y}(\omega) \cos \phi_{x}(\omega)+n_{x}(\omega) \cos \phi_{y}(\omega)}
$$

The reflectance is simply $R(\omega)=|r(\omega)|^{2}$. The computed curve for the part of the intensity, which is absorbed in the sample (this is $(1-R(\omega)$ ) is shown in Figure $4(\mathrm{~d})$. This curve shows that about $20 \%$ of the light is absorbed by the $\sim 5 \mathrm{~nm}$ thick film at $595 \mathrm{~cm}^{-1}$. Curve (c) in Figure 4 is curve (d) multiplied by the FEL beam power (see supplemental material). This introduces some weak modulations, but does not affect the relevant information that the peak maxima of the calculated curves are essentially at the same energy as the maxima in the experimental curves, which together with the temperature rise curve is a strong indication that the desorption process is thermal. The energies of the peak maxima are near to the energy of the longitudinal optical (LO) phonon corresponding to the TO phonon with $\omega_{1}^{T O}=272 \mathrm{~cm}^{-1}$. The LO phonon energy can be calculated with the Lyddane-Sachs-Teller relation [ $\left.\left(\omega^{L O} / \omega^{T O}\right)^{2}=\epsilon_{0} / \epsilon_{\infty}\right]$ to $\omega_{1}^{L O}=585 \mathrm{~cm}^{-1}$. At about this energy also the $\mathrm{CeO}_{2}$ restrahlen band vanishes ${ }^{31}$.

The peak maximum in the action spectrum [Figure 4(a)] is at slightly higher energy than the maxima in all the other curves. One possible explanation is the decrease of the rare gas coverage during the scan, which would shift the maximum to higher energy, as observed. Also experimental uncertainties and issues with theoretical approximations may have to be considered. Additionally we note that the maximum in the temperature rise curve is probably shifted to somewhat lower energy with respect to the real absorption maximum due to the thermic inertia of the system.

In a rather simple picture the sample temperature rise would be about proportional to the absorbed infrared power, and the rare gas desorption rate would be given by the temperature dependent rare gas vapor pressure, which does depend non-linearly on the temperature and the rare gas coverage. However, one aspect not considered is that the FEL laser beam is not continuous, but consists of short pulses, which induce temperature spikes much higher than the average sample temperature. Thus, the relationship between the intensities in the surface action spectra and the absorbed infrared power may be expected to be rather complex. At this point we note that the term 'temperature' may not always well apply to the state of the sample during laser beam irradiation if this term is considered to be related to an equilibrium thermal distribution of energy in the different degrees of freedom.

Apparently, the peak in the action spectrum is much sharper than that in the temperature curve and it is essentially symmetric. One possible explanation for this is the already mentioned non-linear dependence of the vapor pressure on the temperature. This may produce sharper or wider peaks depending on the sublimation energy. However, it is not expected that the asymmetry is completely hidden. Thus, a probably better explanation is that the intense temperature spikes produced by the FEL micropulses are more relevant for the desorption process than the time-integrated average sample temperature measured with the thermopair (averaging time in the range of seconds).

The blue temperature curve in Figure 4 is quite noisy, but it is rather obvious that the peak is asymmetric. This is due to the fact that the rising side of the peaks is determined by the balance of heating (by the FEL infrared beam) and cooling while the slope at the cooldown side is more defined by the cooling power of the cryostat. In the case of the $\mathrm{CeO}_{2}(111) / \mathrm{Ru}(0001)$ spectrum in Figure 4 the scan direction was from high to low energies as indicated in the figure. It can be changed to scan in the other direction to check the hysteresis (not shown here).

\section{B. The direct coupling channel $-\mathrm{V}_{2} \mathrm{O}_{3}(0001) / \mathrm{Au}(111)$}

A thin $\mathrm{V}_{2} \mathrm{O}_{3}(0001)$ layer on $\mathrm{Au}(111)$ was chosen as the test system for the surface sensitivity of the new method. In previous studies it was shown that the $\mathrm{V}_{2} \mathrm{O}_{3}(0001)$ surface is terminated by a layer of vanadyl groups under common ultra high vacuum conditions ${ }^{37-40}$. These groups have a characteristic vibrational energy in the range of $1000 \mathrm{~cm}^{-1}$ and they are only found at the sur- 
face and not in the bulk of the oxide, which means that they can be used to check the surface sensitivity of the surface action spectroscopy method. IRAS and action spectra of $\mathrm{V}_{2} \mathrm{O}_{3}(0001)$, clean and with adsorbates, are shown in Figure 5.

The temperature rise due to IR absorption is shown in curve (d) while curves (b) and (c) display action spectra of the surface recorded with an argon and a neon messenger gas, respectively. Apparently, argon messenger atoms cannot be desorbed by the infrared radiation while this is possible for neon messenger atoms. Deposition of $1 \AA$ of iron [curve (e)] removes the vanadyl vibrational signal, which is an independent proof that this is a surface vibration. Curve (f) shows that adsorbate levels can be detected with the example of a methanol adsorbate (some details of methanol on $\mathrm{V}_{2} \mathrm{O}_{3}(0001)$ have been published by Romanyshyn et $\mathrm{al}^{41}$ and Göbke et $\mathrm{al}^{42}$ ).

The data demonstrate (1) that the action spectroscopy method is surface sensitive enough to detect surfacelocalized vibrations like the vanadyl vibration and adsorbate vibrations and (2) that argon is not suitable as a messenger gas in the present case. An idea why argon is not suitable may be derived from a compilation of desorption energies published by Schlichting ${ }^{43}$ (tables V.I.20 and V.I.21). These tables list desorption energies ranging from $\sim 81 \mathrm{~cm}^{-1}$ to $\sim 1000 \mathrm{~cm}^{-1}$ for neon and from $\sim 660 \mathrm{~cm}^{-1}$ to $\sim 960 \mathrm{~cm}^{-1}$ for argon. The upper value for neon is a singular high value; without consideration of this value the range for neon is $\sim 81 \mathrm{~cm}^{-1}$ to $\sim 299 \mathrm{~cm}^{-1}$. If these values are contrasted with the energy of the vanadyl vibration, $1043 \mathrm{~cm}^{-1}$, see Figure 5(c), then it is clear that a substantial part of this energy would be required to desorb argon, while neon desorption would require much less of the vibrational energy. Since helium is even more weakly bound than neon, it may be assumed that the use of helium messenger atoms would improve the sensitivity of the method further.

At this point of the discussion it is important to get an understanding of the desorption mechanism in the case of surface-localized modes. From Figure 5(d) it is clear that the temperature rise connected with the vanadyl vibration is so small that it could not be detected with the given experimental setup, indicating that the desorption process may be non-thermal. The absorbance of the vanadyl vibration was about $1 \%$ in the IRAS spectrum [curve (a) in Figure 5], which is much less than that of the peak in the $\mathrm{CeO}_{2}(111)$ layer on $\mathrm{Ru}(0001)$ [curve (d) in Figure 4]. From the parameters of the FHI FEL, the diameter of the infrared beam on the sample of $3 \mathrm{~mm}$, and from the vanadyl group absorbance of $1 \%$, it may be estimated that one vanadyl group absorbs $\sim 0.1$ photons per micro pulse. Since the time between two successive micro pulses, 1 nsec, is larger than typical vibrational lifetimes, it follows that the vanadyl groups probably do not reach higher vibrationally excited states than the first one. A higher vibrational state would be hard to reach anyway by sequential photon absorption due to the anharmonicity of the vibration. Evidence for a nonlinear optical

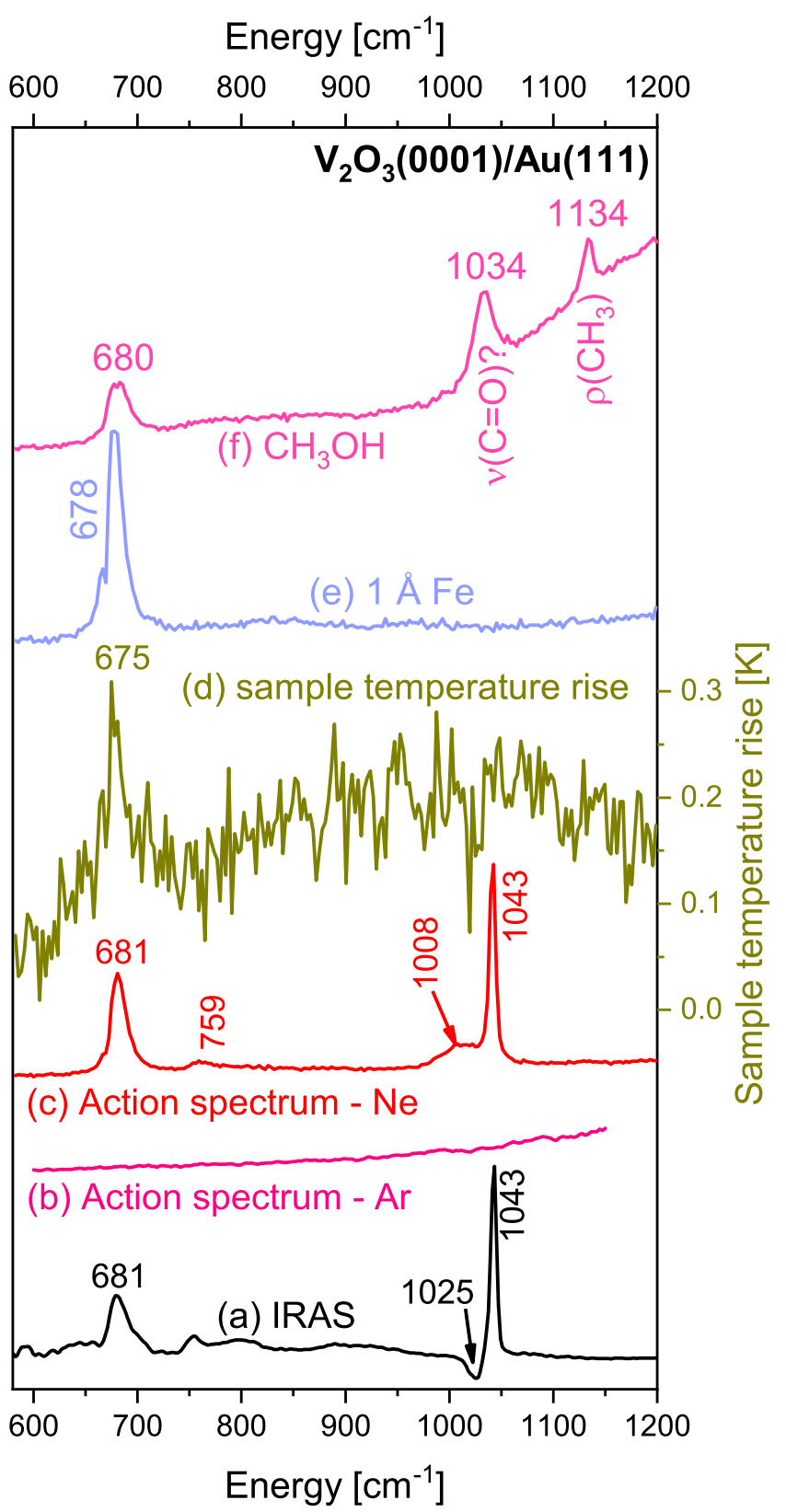

FIG. 5. (color online). Argon and neon [curve (b) and (c)] surface action spectra of $\mathrm{V}_{2} \mathrm{O}_{3}(0001) / \mathrm{Au}(111)$ in comparison with an IRAS spectrum [curve (a)]. Curve (d) displays the sample temperature rise when spectrum (c) was recorded. Curves (e) and (f) were recorded after deposition of iron and dosage of methanol, respectively. A clear identification of the peak at $1034 \mathrm{~cm}^{-1}$ in curve (f) is not possible - it may be due either to the methanol $\mathrm{C}=\mathrm{O}$ or to the vanadyl $\mathrm{V}=\mathrm{O}$ vibration.

process, two photon absorption, could not be found by experiments where the beam intensity was varied. However, these experiments were not fully conclusive since (1) the sample had to be flashed after every single experiment to desorb contaminations, which tends to change the vanadyl vibrational energy and absorption cross section to 
some extent and (2) since there was a notable effect of rare gas depletion on the desorption rate at high beam intensities.

The essence of this discussion is that infrared induced desorption in the case of the vanadyl groups is probably a single photon process. Energy is transferred by direct vibrational coupling from the excited vanadyl vibration to the bond between the rare gas atoms and the surface. The same conclusion does apply to desorption induced by excitation of adsorbate levels, see Figure 5(f). For both type of excitations it is clear that they are surface related.

The action spectra shown in Figure 5 are nonreferenced spectra, i.e. they are not referenced to a reference spectrum. This is different for the IRAS spectrum displayed in Figure 5(a). In this case the displayed curve is the result of the division of two spectra, a spectrum of vanadyl terminated $\mathrm{V}_{2} \mathrm{O}_{3}(0001)$, the sample spectrum, and a spectrum of a surface where the vanadyl groups were removed by electron irradiation, the reference spectrum. If the differences between sample and reference sample are localized in the surface region, then all bulk-related intensity is removed by the division procedure. Electron irradiation leads to an ordered surface without vanadyl groups ${ }^{44}$, but the reduced, vanadyl free surface is very reactive and via reaction with carbon dioxide in the residual gas atmosphere new vanadyl groups are formed ${ }^{45}$. The vibrational energy of these newly formed vanadyl groups is lower due to the lower density of these groups and the consequently reduced dipole coupling. The vibrational band of these groups in the reference spectrum is the reason for the negative intensity at $1025 \mathrm{~cm}^{-1}$, which swamps the band at $1008 \mathrm{~cm}^{-1}$ in Figure 5(c). This latter band is tentatively assigned to vanadyl groups near to surface defects such as missing vanadyl groups. The presence of spectral intensity at critical energies in the reference spectrum is a common, but rarely discussed obstacle of IRAS. Surface action spectroscopy does not have this issue.

Finally the level at $\sim 680 \mathrm{~cm}^{-1}$ in Figure 5 shall be discussed. Curve (d) shows a clear temperature rise at about this energy, which indicates that this level is probably not surface-localized. According to literature bulk-related intensity may be expected between 600 and $700 \mathrm{~cm}^{-121,46}$.

\section{SUMMARY}

We have set up an apparatus for surface action spectroscopy with rare gas messenger atoms. It could be shown that the method is sensitive to surface-localized vibrations, where probably just a single photon induces desorption of a rare gas atom. Rare gas desorption may also be induced by excitation of bulk-related states. In this case a substantial sample temperature rise is detected, which leads to quasi-thermal rare gas desorption. In a more general picture one may state: the more the excited vibrational state extends into the sample volume, the more energy is required to induce desorption.
The action spectrum of $\mathrm{V}_{2} \mathrm{O}_{3}(0001) / \mathrm{Au}(111)$ is very similar to the IRAS spectrum, but contrary to IRAS, action spectroscopy does not require spectra of reference samples. Features in reference spectra can swamp spectral features as shown for the $\mathrm{V}_{2} \mathrm{O}_{3}(0001)$ case.

Future development will go into two direction. One direction is the implementation of helium as a messenger gas. Due to its very weak interaction with the sample this messenger gas will affect the sample vibrational properties more weakly than neon and it may be expected that the use of helium will increase the sensitivity of the method since less energy is required to desorb it. The other direction is the setup of a sample holder with a good temperature control at cryogenic temperatures. This would permit to use selective decoration of features at the sample surface as a spectroscopic tool. An oxide surface decorated with metal clusters, a model catalyst, may serve as an example. Here it may be possible by proper choice of the adsorption temperature to decorate just the clusters with rare gas atoms. In this case the action spectroscopy signal would come exclusively from the clusters, which may be exploited to get a vibrational spectrum of just the deposited aggregates. This is probably the most promising application of the surface action spectroscopy method.

\section{SUPPLEMENTARY MATERIAL}

See Supplementary Material for a plot of the beam power as a function of the photon energy for the spectra shown in Figure 4.

\section{ACKNOWLEDGMENTS}

This work was supported by the Fonds der Chemischen Industrie. Zongfang Wu thanks the Alexander von Humboldt foundation (AvH) for supporting her with an $\mathrm{AvH}$ research grant.

${ }^{1}$ B. Simard, S. Denommee, D. Rayner, D. van Heijnsbergen, G. Meijer, and G. von Helden, Chem. Phys. Lett. 357, 195 (2002).

${ }^{2}$ M. Brümmer, C. Kaposta, G. Santambrogio, and K. R. Asmis, J. Chem. Phys 119, 12700 (2003).

${ }^{3}$ A. Fielicke, G. Meijer, and G. von Helden, J. Am. Chem. Soc. 125, 3659 (2003).

${ }^{4}$ D. van Heijnsbergen, K. Demyk, M. A. Duncan, G. Meijer, and G. von Helden, Phys. Chem. Chem. Phys. 5, 2515 (2003).

${ }^{5}$ A. Fielicke, A. Kirilyuk, C. Ratsch, J. Behler, M. Scheffler, G. von Helden, and G. Meijer, Phys. Rev. Lett. 93, 023401 (2004).

${ }^{6}$ A. Fielicke, C. Ratsch, G. von Helden, and G. Meijer, J. Chem. Phys. 122, 091105 (2005).

${ }^{7}$ A. Fielicke, G. von Helden, G. Meijer, B. Simard, and D. M. Rayner, J. Phys. Chem. B 109, 23935 (2005).

${ }^{8}$ P. Gruene, D. M. Rayner, B. Redlich, A. F. G. van der Meer, J. T. Lyon, G. Meijer, and A. Fielicke, Science 321, 674 (2008).

${ }^{9}$ I. Swart, F. M. F. de Groot, B. M. Weckhuysen, P. Gruene, G. Meijer, and A. Fielicke, J. Phys. Chem. A 112, 1139 (2008).

${ }^{10}$ R. Gehrke, P. Gruene, A. Fielicke, G. Meijer, and K. Reuter, J. Chem. Phys. 130, 034306 (2009). 
${ }^{11}$ L. Lin, P. Claes, T. Hoeltzl, E. Janssens, T. Wende, R. Bergmann, G. Santambrogio, G. Meijer, K. R. Asmis, M. T. Nguyen, and P. Lievens, Phys. Chem. Chem. Phys. 12, 13907 (2010).

${ }^{12}$ M. Haertelt, V. J. F. Lapoutre, J. M. Bakker, B. Redlich, D. J. Harding, A. Fielicke, and G. Meijer, J. Phys. Chem. Lett. 2, $1720(2011)$.

${ }^{13}$ A. P. Woodham, G. Meijer, and A. Fielicke, Angew. Chem., Int. Ed. 51, 4444 (2012).

${ }^{14}$ A. P. Woodham, G. Meijer, and A. Fielicke, J. Am. Chem. Soc. 135, 1727 (2013)

${ }^{15}$ M. R. Fagiani, X. Song, P. Petkov, S. Debnath, S. Gewinner, W. Schöllkopf, T. Heine, A. Fielicke, and K. R. Asmis, Angew. Chem., Int. Ed. 56, 501 (2017).

${ }^{16}$ K. R. Asmis, Phys. Chem. Chem. Phys. 14, 79270 (2012).

${ }^{17}$ H. Kuhlenbeck, S. Shaikhutdinov, and H.-J. Freund, Chem. Rev. 113, 3986 (2013).

${ }^{18}$ Z. Wu, A. Plucienik, F. E. Feiten, M. Naschitzki, W. Wachsmann, S. Gewinner, W. Schöllkopf, V. Staemmler, H. Kuhlenbeck, and H.-J. Freund, Phys. Rev. Lett. 119, 136101 (2017).

${ }^{19}$ H. Schlichting and D. Menzel, Rev. Sci. Instrum. 64, 2013 (1993).

${ }^{20}$ W. Schöllkopf, S. Gewinner, H. Junkes, A. Paarmann, G. von Helden, H. Bluem, and A. M. M. Todd, Proc. SPIE 9512 , 95121L (2015).

${ }^{21}$ M. Abu Haija, S. Guimond, Y. Romanyshyn, A. Uhl, H. Kuhlenbeck, T. K. Todorova, M. V. Ganduglia-Pirovano, J. Döbler, J. Sauer, and H.-J. Freund, Surf. Sci. 600, 1497 (2006).

${ }^{22}$ X. Song, E. Primorac, H. Kuhlenbeck, and H.-J. Freund, Surf. Sci. 653, 181 (2016).

${ }^{23}$ M. Baron, O. Bondarchuk, D. Stacchiola, S. Shaikhutdinov, and H.-J. Freund, J. Phy. Chem. C 113, 6042 (2009).

${ }^{24}$ J.-L. Lu, H.-J. Gao, S. Shaikhutdinov, and H.-J. Freund, Surf. Sci. 600, 5004 (2006)

${ }^{25}$ D. R. Mullins, Surf. Sci. Rep. 70, 42 (2015).

${ }^{26}$ D. R. Mullins, P. V. Radulovic, and S. H. Overbury, Surf. Sci. 429, 186 (1999).

${ }^{27}$ S. Eck, C. Castellarin-Cudia, S. Surnev, M. G. Ramsey, and F. P. Netzer, Surf. Sci. 520, 173 (2002).

${ }^{28}$ Y. J. Chabal, Surface Science Reports 8, 211 (1988).

${ }^{29}$ Y. Wang and C. Wöll, Chem. Soc. Rev. 46, 1875 (2017).
${ }^{30}$ F. Marabelli and P. Wachter, Phys. Rev. B 36, 1238 (1987).

${ }^{31}$ S. Mochizuki and S. Tateyama, Phys. Stat. Sol. (b) 110, K1 (1982).

${ }^{32}$ S. Mochizuki, phys. stat. sol. (b) 114, 189 (1982).

${ }^{33}$ B. G. Frederick, G. Apai, and T. N. Rhodin, Phys. Rev. B 44 1880 (1991).

${ }^{34}$ R. L. Olmon, B. Slovick, T. W. Johnson, D. Shelton, S.-H. Oh, G. D. Boreman, and M. B. Raschke, Phys. Rev. B 86, 235147 (2012).

${ }^{35}$ M. Born and E. Wolf, Principles of Optics, $4^{\text {th }}$ ed. (Pergamon Press, London, 1970).

${ }^{36}$ O. S. Heavens, Rep. Prog. Phys. 23, 1 (1960).

${ }^{37}$ F. E. Feiten, J. Seifert, J. Paier, H. Kuhlenbeck, H. Winter, J. Sauer, and H.-J. Freund, Phys. Rev. Lett. 114, 216101 (2015).

${ }^{38}$ F. E. Feiten, H. Kuhlenbeck, and H.-J. Freund, J. Phys. Chem. C 119, 22961 (2015).

${ }^{39}$ J. Schoiswohl, M. Sock, S. Surnev, M. G. Ramsey, F. P. Netzer, G. Kresse, and J. N. Andersen, Surf. Sci. 555, 101 (2004).

${ }^{40}$ A.-C. Dupuis, M. Abu Haija, B. Richter, H. Kuhlenbeck, and H.-J. Freund, Surf. Sci. 539, 99 (2003).

${ }^{41}$ Y. Romanyshyn, S. Guimond, D. Göbke, J. M. Sturm, H. Kuhlenbeck, J. Döbler, M. V. Ganduglia-Pirovano, J. Sauer, and H.-J. Freund, Top. Catal. 54, 669 (2011).

${ }^{42}$ D. Göbke, Y. Romanyshyn, S. Guimond, J. M. Sturm, H. Kuhlenbeck, J. Döbler, U. Reinhardt, M. V. GandugliaPirovano, J. Sauer, and H.-J. Freund, Angew. Chem. Int. Ed. 48, 3695 (2009).

${ }^{43} \mathrm{H}$. Schlichting, Methoden und Mechanismen der thermischen Desorption: Adsorptions-, Desorptions-Kinetik, Epitaxie und Ordnung von Edelgasschichten auf Ru(001)., Ph.D. thesis, Technische Universität München, München, Germany (1990).

${ }^{44}$ F. E. Feiten, H. Kuhlenbeck, and H.-J. Freund, PCCP 18, 3124 (2016).

${ }^{45}$ M. Abu Haija, Y. Romanyshyn, A. Uhl, H. Kuhlenbeck, and H. J. Freund, Top. Catal. 60, 413 (2017).

${ }^{46}$ G. Kresse, S. Surnev, J. Schoiswohl, and F. P. Netzer, Surf. Sci. 555, 118 (2004). 